\title{
Morphology and Growth Kinetics of Hyphae of Differentiated and Undifferentiated Mycelia of Neurospora crassa
}

\author{
By G. C. STEELE AND A. P. J. TRINCI \\ Microbiology Department, Queen Elizabeth College, University of London, \\ London $W 87 A H$
}

(Received I5 April I975; revised I6 June 1975)

\begin{abstract}
SU MM A R Y
A comparison was made of the morphology and growth kinetics of hyphae of differentiated and undifferentiated mycelia of Neurospora crassa. Undifferentiated mycelia were formed during exponential growth on solid media or submerged culture. Hyphae at the margin of differentiated mycelia (colonies) differed from undifferentiated mycelia in diameter, extension rate, extension zone length, and intercalary and apical compartment length. The mean hyphal extension rate $(E)$ of an undifferentiated mycelium was a function of the length of the mycelium's hyphal growth unit $(G)$ and the organism's specific growth rate $(\alpha)$. Thus, $E=G \alpha$.
\end{abstract}

\section{INTRODUCTION}

Many changes occur as a fungal spore develops into an undifferentiated mycelium and then into a 'mature', differentiated colony. Undifferentiated mycelia are produced under the constant or near constant conditions which prevail during exponential growth on solid media (Trinci, 1974) or in submerged culture. Hyphae at the margin of differentiated mycelia (colonies) grow under conditions which change relatively rapidly as colonization of a specific region of the medium proceeds. The hyphae of these two types of mycelia differ in morphology and growth kinetics. Most studies of the morphology and growth of individual hyphae have been made upon hyphae at the margin of colonies. However, the basic features of fungal growth are likely to be obscured in such hyphae by the differentiation process.

The present study was undertaken to establish the morphological and kinetic characteristics which distinguish hyphae of differentiated and undifferentiated mycelia. In addition, it was hoped to determine the degree of similarity between undifferentiated mycelia formed on solid media and in submerged culture.

Total hyphal length and number of tips of an undifferentiated mycelium increase exponentially at the same specific growth rate (Trinci, 1974). Growth of such mycelia thus involves the exponential duplication of a hypothetical 'hyphal growth unit', $G$ (defined as the total hyphal length of the mycelium divided by its number of tips), which, unlike an individual cell of a unicellular micro-organism, is a physiological but not a morphological entity (Trinci, 1974). The mean hyphal extension rate, $E$ (in $\mu \mathrm{m} / \mathrm{tip} / \mathrm{h}$ ), of an undifferentiated mycelium may be determined (Trinci, 1974) from the equation

$$
E=\frac{2\left(H_{\mathrm{t}}-H_{\mathrm{o}}\right)}{B_{\mathrm{o}}+B_{\mathrm{t}}},
$$

where $H_{\mathrm{o}}$ is the total hyphal length at zero time, $H_{\mathrm{t}}$ the total hyphal length $\mathrm{I} \mathrm{h}$ later, $B_{\mathrm{o}}$ the number of hyphal tips at zero time, and $B_{\mathrm{t}}$ the number of hyphal tips $\mathrm{I} h$ later. The extension 
rate, $K_{\mathrm{r}}$, of a colony (or of a hypha) is a function of the width (length) of its peripheral growth zone, $w$, and the organism's specific growth rate, $\alpha$ (Trinci, 1971). Thus,

$$
K_{\mathrm{r}}=w \alpha .
$$

It follows from eqn 2 that the mean hyphal extension rate of an undifferentiated mycelium will be a function of the length of its hyphal growth unit, $G$, and its specific growth rate, $\alpha$, giving

$$
E=G \alpha .
$$

Since $G$ (except during the early stages of growth; Trinci, 1974) and $\alpha$ are constants, $E$ must also be a constant. $E$ has in fact been shown to be a constant for undifferentiated mycelia of Geotrichum candidum having more than about three tips (Trinci, 1974). The validity of eqn 3 may be established by comparing the values for $E$ obtained using eqns I and 3. However, eqn I would be expected to over-estimate $E$ because branches are initiated exponentially (Trinci, 1974).

\section{METHODS}

Organisms and culture media. The Neurospora crassa mutants spco-9 (spreading colonial) and $\cot -3$ (colonial temperature-sensitive) were obtained from the Fungal Genetics Stock Center, Humboldt State College Foundation, Arcata, California, U.S.A. These strains were chosen for this study because they grow filamentously in batch culture. Neurospora crassa cot-3 has wild-type characteristics at $25{ }^{\circ} \mathrm{C}$.

The strains were grown on Vogel's (1956) minimal medium with $\mathrm{I} \%(\mathrm{w} / \mathrm{v})$ sucrose as the carbon source. For the preparation of agar plates, the medium was gelled with Oxoid 'purified' agar (final concentration $\mathrm{I} \cdot 5 \% \mathrm{w} / \mathrm{v}$ ). Batch cultures were grown in $250 \mathrm{ml}$ flasks containing $25 \mathrm{ml}$ medium. Cultures were inoculated and incubated at $25{ }^{\circ} \mathrm{C}$ on a rotary shaker (200 rev./min) as described previously (Trinci, I969). Petri dishes $(9 \mathrm{~cm})$ containing $20 \mathrm{ml}$ medium were overlaid with sterile discs of cellophane (PT 300, British Cellophane Ltd). Conidia were inoculated on to the cellophane, thus ensuring that growth was in a single plane. Undifferentiated mycelia and colonies were both grown on solid media overlaid with cellophane. All cultures were incubated at $25{ }^{\circ} \mathrm{C}$.

Measurements of hyphal characteristics. The $G$ values of undifferentiated mycelia grown on solid media and in submerged culture were determined as described previously (Trinci, 1974). The $E$ values were determined using eqn I as described previously (Trinci, 1974). Peripheral growth zone lengths of hyphae of undifferentiated mycelia were determined by the method described by Trinci (1974). The diameters of hyphae were measured using a travelling-eyepiece micrometer.

Hyphal length specific growth rates of undifferentiated mycelia were determined by timelapse photography as described previously (Trinci, 1974). Biomass specific growth rates in batch culture were determined as described previously (Trinci, 1969). The strains grew in a filamentous form in submerged culture.

The mean lengths of the tapered regions of hyphal tips (i.e. the extension zone lengths; Trinci \& Halford, 1975) were determined by photographing hyphal tips at a magnification of $\times$ I500 using a Nikon 'Apophot' photomicroscope and subsequently measuring enlarged tracings of these tips with the aid of a Shadowmaster (Pirt, 1967).

The radial growth rates of colonies growing on media overlaid with sterile cellophane were determined as described previously (Trinci, 1974). The extension rate of the 'leading' hyphae of colonies and their primary and secondary branches were determined by timelapse photography. 


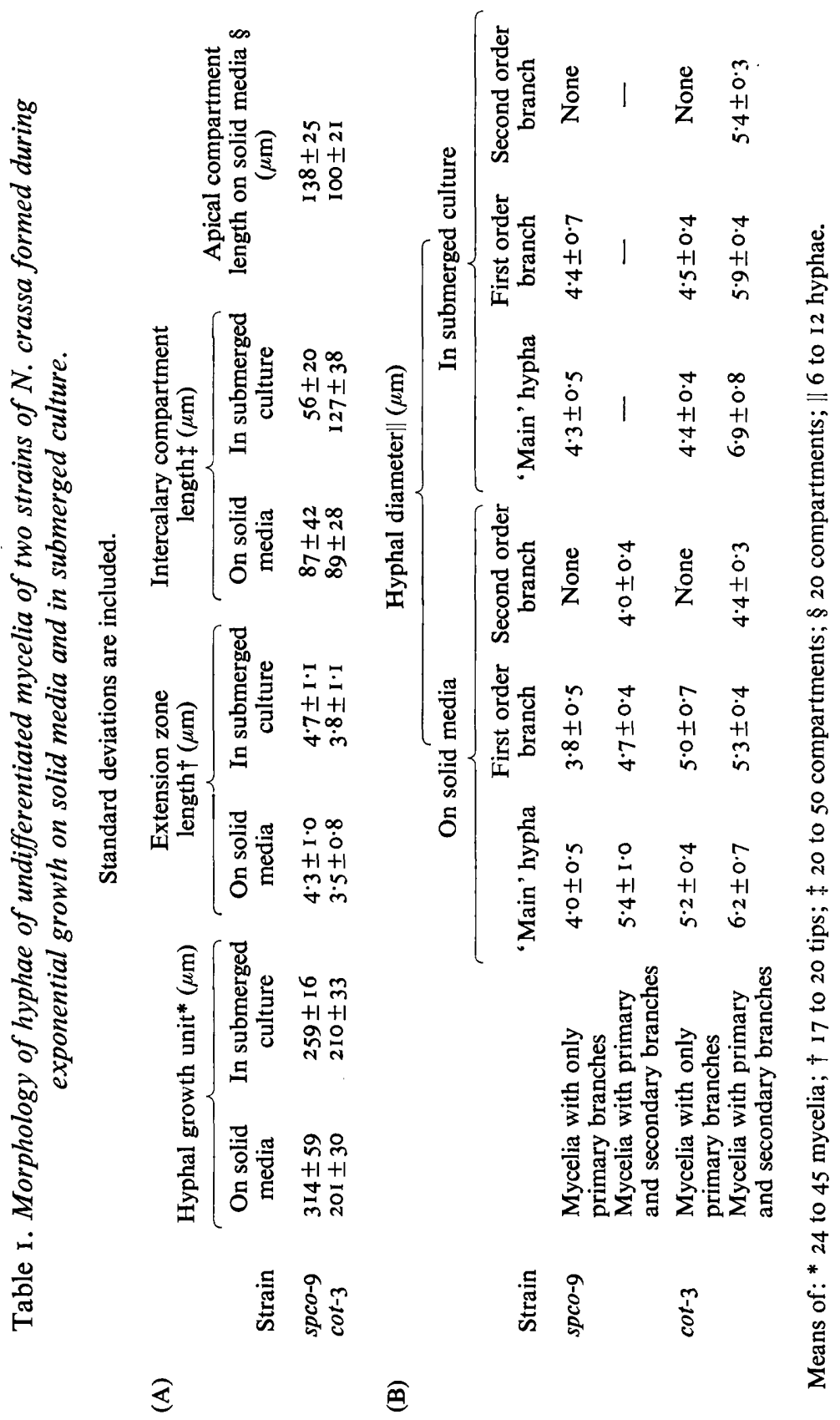




\section{RESULTS}

Comparison of the growth kinetics and morphology of undifferentiated mycelia grown on solid media and in submerged culture

The morphology and growth kinetics of undifferentiated mycelia of $N$. crassa cultured on solid media and in submerged culture are compared in Tables $I$ and 2 and Fig. 2. The measurements were made on $24 \mathrm{~h}$ cultures of $N$. crassa spco-9, and 24 and $48 \mathrm{~h}$ cultures of $N$. crassa cot -3 . There was a close similarity between the morphology of mycelia cultured on solid media and in submerged culture. Growth was exponential under both cultural conditions. The biomass specific growth rates obtained in submerged culture were less than the hyphal length specific growth rates determined on solid media (Table 2). There was no apparent difference in hyphal growth unit length between mycelia grown on solid media and in submerged culture (Table IA). In young mycelia, before the initiation of secondary branches, there was no apparent difference between the diameter of 'main' and primary branches (Table IB). In older mycelia the secondary branches appeared to be slightly narrower than the 'main' hyphae.

The $E$ values of undifferentiated mycelia grown on solid media were determined using eqns I and 3. The similarity between the values for $E$ obtained by the direct (eqn I) and indirect (eqn 3) methods (see Table 2) substantiates the validity of eqn 3, viz. the mean hyphal extension rate of an undifferentiated mycelium is a function of the mean length of hypha per tip (i.e. the hyphal growth unit length) and the organism's specific growth rate.

\section{Comparison of the growth kinetics and morphology of hyphae of undifferentiated and differentiated mycelia of $N$. crassa}

The hyphae at the margin of 'mature' colonies of $N$. crassa were differentiated into wide 'leading' hyphae and narrower branch hyphae (Table 3 and Fig. 3). The maximum extension rates $\left(E_{\max }\right)$ of the 'leading' hyphae of differentiated mycelia were about twofold (spco-9) to about sevenfold ( $\cot -3)$ faster than the maximum extension rates of hyphae of undifferentiated mycelia (Tables 2 and 3). Hyphae of undifferentiated mycelia had maximum peripheral growth zone lengths of about $2000 \mu \mathrm{m}$ (spco-9) and about $1450 \mu \mathrm{m}$ ( $\cot -3)$. The 'leading' hyphae of 'mature' colonies had longer extension zones (Fig. I), apical compartments and intercalary compartments than the hyphae of undifferentiated mycelia (Tables IA and 3).

The main characteristics of hyphae of differentiated and undifferentiated mycelia of $N$. crassa are contrasted in Table 4.

Table 2. Growth kinetics of undifferentiated mycelia of two strains of $N$. crassa found during exponential growth on solid media and in submerged culture

Standard deviations are included.

\begin{tabular}{|c|c|c|c|c|c|}
\hline \multirow[b]{2}{*}{ Strain } & \multicolumn{2}{|c|}{ Specific growth rate, $\alpha^{*}\left(\mathrm{~h}^{-1}\right)$} & \multicolumn{2}{|c|}{$\begin{array}{l}\text { Mean hyphal extension } \\
\text { rate, } E \dagger(\mu \mathrm{m} / \mathrm{tip} / \mathrm{h}) \text {, } \\
\text { calculated using: }\end{array}$} & \multirow{2}{*}{$\begin{array}{c}\text { Approximate } \\
\text { maximum hyphal } \\
\text { extension rate, } \\
E_{\max } \dagger(\mu \mathrm{m} / \mathrm{tip} / \mathrm{h})\end{array}$} \\
\hline & $\begin{array}{l}\text { Hyphal length } \\
\text { on solid media }\end{array}$ & $\begin{array}{l}\text { Biomass in sub- } \\
\text { merged culture }\end{array}$ & Eqn I & Eqn 3 & \\
\hline spco-9 & $0.36 \pm 0.04$ & $0.22 \pm 0.03$ & 95 & 84 & 443 \\
\hline $\cot -3$ & $0.21 \pm 0.02$ & $0.15 \pm 0.03$ & 59 & 42 & 125 \\
\hline
\end{tabular}

\footnotetext{
* Mean of 4 observations. $\dagger$ Measurements made on mycelia growing on solid media; the hyphal length
} specific growth rate was used in eqn 3 . 
(a)

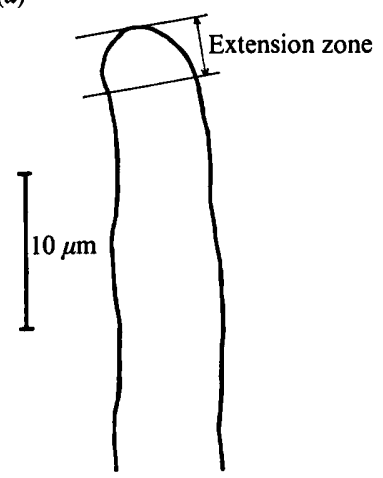

(b)

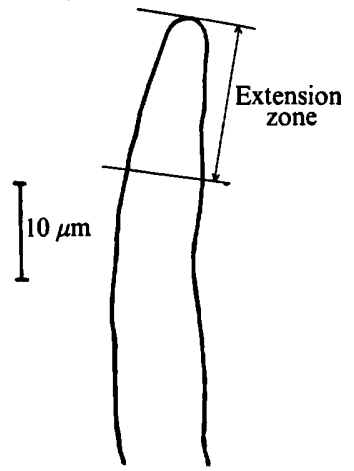

Fig. I

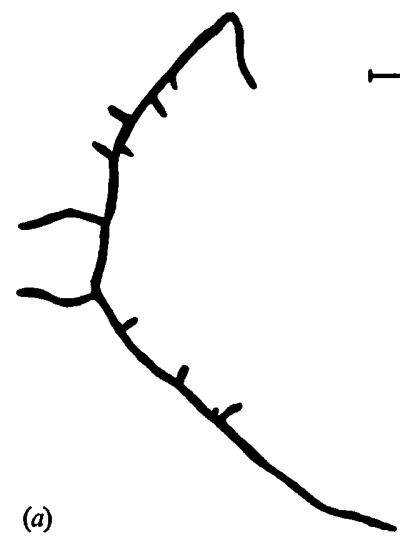

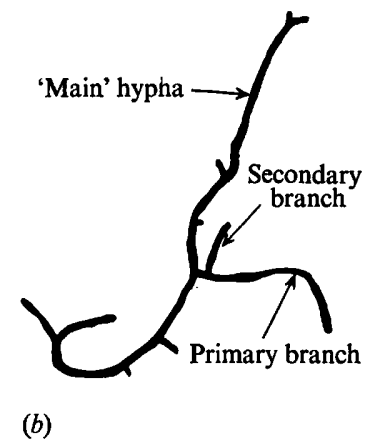

$500 \mu \mathrm{m}$

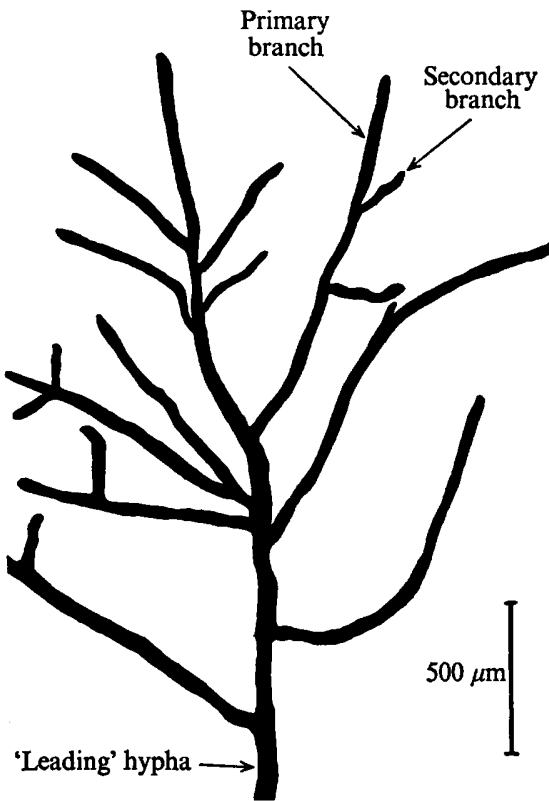

Fig. 3

Fig. 2

Fig. I. (a) Tip of a 'main' hypha of an undifferentiated mycelium of $N$. crassa cot-3, 24 h after inoculation on to solid medium. (b) Tip of a 'leading' hypha at the margin of a 'mature' colony of $N$. crassa cot -3 on solid medium.

Fig. 2. (a) An undifferentiated mycelium of $N$. crassa spco-9 grown on solid medium, $24 \mathrm{~h}$ after inoculation. (b) An undifferentiated mycelium of $N$. crassa spco-9 grown in submerged culture, $18 \mathrm{~h}$ after inoculation.

Fig. 3. A hyphal system at the margin of a 'mature' colony of $N$. crassa cot-3.

\section{DISCUSSION}

On germination, a fungal spore develops into an undifferentiated mycelium which increases in size and gradually differentiates into a 'mature' colony (Yanagita \& Kogane, 1962). Hyphae at the margin of colonies are usually, but not always, differentiated into'wide 'leading' hyphae and narrower branch hyphae (Trinci, 1973). This differentiation probably occurs at least in part as a response to the chemical changes induced in the medium by growth of the mould (Park \& Robinson, 1966). The fast radial growth rate of some fungal colonies is clearly associated with the production of these leading hyphae (Trinci, 1974; Tables 2 and 3). Hyphae of undifferentiated mycelia of $N$. crassa have a lower $E_{\max }$ than the leading hyphae at the margin of differentiated colonies (Tables 2 and 3). However, in some species (e.g. Geotrichum candidum) the $E_{\max }$ of hyphae of undifferentiated mycelia is very similar to the radial growth rate of their differentiated colonies (Trinci, 1974). 
Table 3. Morphology and growth kinetics of hyphae at the margin of differentiated colonies of two strains of $N$. crassa

Standard deviations are included. Growth rates, expressed as percentages of the growth rate of the 'leading' hyphae, are given in parentheses.

\begin{tabular}{|c|c|c|c|}
\hline $\begin{array}{l}\text { Diameter of hyphae } \\
\text { at the colony's } \\
\text { margin* }(\mu \mathrm{m})\end{array}$ & $\begin{array}{l}\text { 'Leading' hyphae } \\
\text { Primary branches } \\
\text { Secondary branches }\end{array}$ & $\begin{array}{l}s p c o-9 \\
\mathrm{II} \cdot 7 \pm 0 \cdot 6 \\
7 \cdot 0 \pm \mathrm{I} \cdot 0 \\
5 \cdot 7 \pm 0 \cdot 7\end{array}$ & $\begin{aligned} c o t-3 \\
10 \cdot 5 \pm 1 \cdot 6 \\
6 \cdot 3 \pm 0 \cdot 7 \\
5 \cdot 1 \pm 0 \cdot 7\end{aligned}$ \\
\hline $\begin{array}{l}\text { Compartment length } \\
\text { of 'leading' hyphae } \dagger \\
(\mu \mathrm{m})\end{array}$ & $\begin{array}{l}\text { Apical } \\
\text { Intercalary }\end{array}$ & $\begin{array}{l}419 \pm 110 \\
149 \pm 83\end{array}$ & $\begin{array}{r}309 \pm 52 \\
99 \pm 42\end{array}$ \\
\hline $\begin{array}{l}\text { Extension zone } \\
\text { length of 'leading' } \\
\text { hyphae } \ddagger(\mu \mathrm{m})\end{array}$ & & $18 \cdot 6 \pm 2 \cdot 9$ & $15 \cdot 4 \pm 3 \cdot 2$ \\
\hline $\begin{array}{l}\text { Colony radial growth } \\
\text { rate, } K_{\mathrm{r}} \S(\mu \mathrm{m} / \mathrm{h})\end{array}$ & & 988 & 885 \\
\hline $\begin{array}{l}\text { Linear growth } \\
\text { rates of hyphae } \\
\text { at the margin of } \\
\text { the colony, } \\
E_{\max } !(\mu \mathrm{m} / \mathrm{tip} / \mathrm{h})\end{array}$ & $\begin{array}{l}\text { 'Leading' hyphae } \\
\text { Primary branches } \\
\text { Secondary branches }\end{array}$ & $\begin{array}{c}1020(100 \%) \\
852(83 \%) \\
396(39 \%)\end{array}$ & $\begin{array}{l}942(100 \%) \\
510(55 \%) \\
234(25 \%)\end{array}$ \\
\hline
\end{tabular}

Means of: * I 2 hyphae; $\dagger$ at least 20 compartments; $\ddagger$ I 2 tips; $\S 30$ colonies; $\| 4$ to I I hyphae.

Table 4. A comparison between the characteristics of hyphae of differentiated and undifferentiated mycelia of $N$. crassa

Hyphae formed during exponential growth on solid media or submerged culture

The hyphae of a single mycelium have more or less the same diameter

Each hypha of a mycelium has the same $E_{\max }$

Hyphae have relatively short extension zones

Hyphae do not usually branch sub-apically
Hyphae formed at the margin of differentiated colonies on solid media

The hyphae are differentiated into wide 'leading' hyphae and narrower branch hyphae

'Leading' hyphae have faster $E_{\max }$ values than first and second order branches

'Leading' hyphae have relatively long extension zones

'Leading' hyphae often branch sub-apically

We have established that there is a close morphological similarity between undifferentiated mycelia grown on solid media and in submerged culture. This similarity almost certainly reflects a similarity in their growth kinetics. Thus, results obtained from studies of undifferentiated mycelia on solid media (Trinci, 1974) are almost certainly relevant to growth in submerged culture.

The slight increase in the diameter of hyphae of undifferentiated mycelia with age (Table IA) suggests that there may be a continuous process of mycelial differentiation. Alternatively, ưndifferentiated mycelia may, at least in some species, form a distinct morphological and physiological stage of development. Certainly, it appears that some basic processes of fungal growth are more clearly displayed in undifferentiated mycelia (Trinci, 1974) than in hyphae at the margin of 'mature' colonies. 
We thank the Science Research Council and the Central Research Fund of London University for their financial support.

\section{REFERENCES}

PaRK, D. \& RoBinson, P. M. (1966). Aspects of hyphal morphogenesis in fungi. In Trends in Plant Morphogenesis, pp. 27-44. Edited by E. C. Cutter. London: Longmans, Green.

PIRT, S. J. (1967). A kinetic study of the mode of growth of surface colonies of bacteria and fungi. Journal of General Microbiology 47, I8I-197.

TRINCI, A. P. J. (1969). A kinetic study of the growth of Aspergillus nidulans and other fungi. Journal of General Microbiology 57, I I-24.

TRINCI, A. P. J. (197I). Influence of the peripheral growth zone on the radial growth rate of fungal colonies. Journal of General Microbiology 67, 325-344.

TRINCI, A. P. J. (1973). The hyphal growth unit of wild-type and spreading colonial mutants of Neurospora crassa. Archiv für Mikrobiologie 91, I27-1 36.

TrINCI, A. P. J. (I974). A study of the kinetics of hyphal extension and branch initiation of fungal mycelia. Journal of General Microbiology 8r, 225-236.

Trinci, A. P. J. \& Halford, E. (I975). The extension zone of stage I sporangiophores of Phycomyces blakesleeanus. New Phytologist 74, 8I-83.

VoGEL, H. J. (1956). A convenient medium for Neurospora (medium N). Microbial Genetics Bulletin r3, $42-44$.

YANAGITA, T. \& Kogane, F. (1962). Growth and cytochemical differentiation of mold colonies. Journal of General and Applied Microbiology 8, 20I-213. 